\title{
Lattice QCD determination of patterns of excited baryon states
}

\author{
Subhasish Basak, ${ }^{1}$ R. G. Edwards, ${ }^{2}$ G. T. Fleming, ${ }^{3}$ K. J. Juge, ${ }^{4}$ A. Lichtl, ${ }^{5}$ C. Morningstar, ${ }^{6}$ D. G. Richards, ${ }^{2}$ \\ I. Sato, ${ }^{7}$ and S. J. Wallace ${ }^{8}$ \\ ${ }^{1}$ Department of Physics, Indiana University, Bloomington, Indiana 47405, USA \\ ${ }^{2}$ Thomas Jefferson National Accelerator Facility, Newport News, Virginia 23606, USA \\ ${ }^{3}$ Yale University, New Haven, Connecticut 06520, USA \\ ${ }^{4}$ Department of Physics, University of the Pacific, 3601 Pacific Avenue Stockton, California 95211, USA \\ ${ }^{5}$ RIKEN BNL Research Center Building 510A, Physics Department, Brookhaven National Laboratory, \\ Upton, New York 11973-5000, USA \\ ${ }^{6}$ Department of Physics, Carnegie Mellon University, Pittsburgh, Pennsylvania 15213, USA \\ ${ }^{7}$ Lawrence Berkeley Laboratory, Berkeley, California 94720, USA \\ ${ }^{8}$ University of Maryland, College Park, Maryland 20742, USA \\ (Received 6 August 2007; published 25 October 2007)
}

\begin{abstract}
Energies for excited isospin $I=\frac{1}{2}$ and $I=\frac{3}{2}$ states that include the nucleon and $\Delta$ families of baryons are computed using quenched, anisotropic lattices. Baryon interpolating field operators that are used include nonlocal operators that provide $G_{2}$ irreducible representations of the octahedral group. The decomposition of spin $\frac{5}{2}$ or higher spin states is realized for the first time in a lattice QCD calculation. We observe patterns of degenerate energies in the irreducible representations of the octahedral group that correspond to the subduction of the continuum spin $\frac{5}{2}$ or higher. The overall pattern of low-lying excited states corresponds well to the pattern of physical states subduced to the irreducible representations of the octahedral group.
\end{abstract}

DOI: 10.1103/PhysRevD.76.074504

PACS numbers: $12.38 . \mathrm{Gc}$

\section{INTRODUCTION}

The theoretical determination of the spectrum of baryon resonances from the fundamental quark and gluon degrees of freedom is an important goal for lattice QCD. To date there have been many lattice studies of ground state energies for different baryons [1-3] but only a few results for excited state energies have been reported [4-11]. No clear determination of states with spin $\frac{5}{2}$ or higher has been published because nonlocal operators have not been used. In this work we find degenerate energies that occur in irreducible representations of the octahedral group corresponding to the subduction of the continuum spin $\frac{5}{2}$ or higher. The pattern of lattice QCD baryon states that we observe is compared with the physical spectrum.

Lattice correlation functions correspond to definite irreducible representations (irreps) of the octahedral group when the source and sink operators transform accordingly. There are six double-valued irreps of the octahedral group: three for even parity that are labeled with a $g$ subscript (gerade) and three for odd parity that are labeled with a $u$ subscript (ungerade). They are $G_{1 g}, H_{g}, G_{2 g}, G_{1 u}, H_{u}$, and $G_{2 u}$.

Continuum values of total angular momenta are realized in lattice simulations by patterns of degenerate energies in the continuum limit that match the patterns in Table I for the subduction of spin $J$ to the double-valued irreps of the octahedral group. For example, a state in one of the $G_{2}$ irreps is a signal for the subduction of continuum spin $\frac{5}{2}$ or higher. For spin $\frac{5}{2}$, there must be partner states in the $H$ and $G_{2}$ irreps that would be degenerate in the continuum limit.
For $\operatorname{spin} \frac{7}{2}$, there must be partner states in the $G_{1}, H$, and $G_{2}$ irreps.

This paper reports on work to determine the pattern of low-lying states in the $I=\frac{1}{2}$ and $I=\frac{3}{2}$ channels. We carry out an analysis in quenched lattice QCD using quasilocal and one-link-displaced operators formed from three quark fields. Smeared quark and gluon fields are used $[12,13]$. Smearing reduces the couplings to short wavelength fluctuations of the theory and provides cleaner determinations of energies [14]. This is important when a large array of interpolating field operators is used in order to implement the variational method of Refs. $[15,16]$.

Both quasilocal and nonlocal operators transforming according to the $G_{2}$ irrep are required. We have developed sets of baryon operators that transform according to irreducible representations in two publications, one using an analytical method based on appropriate Clebsch-Gordan coefficients for the octahedral group [17] and the other using a projection method that has been automated and provides very large sets of operators [18]. Results obtained from both methods are found to agree. In this work we use,

TABLE I. The number of occurrences of double-valued irrep $\Lambda$ of the octahedral group for half-integer values of continuum $\operatorname{spin} J$.

\begin{tabular}{lcccccc}
\hline \hline$\Lambda$ & $\mathrm{J}=1 / 2$ & $3 / 2$ & $5 / 2$ & $7 / 2$ & $9 / 2$ & $11 / 2$ \\
\hline$G_{1}$ & 1 & 0 & 0 & 1 & 1 & 1 \\
$H$ & 0 & 1 & 1 & 1 & 2 & 2 \\
$G_{2}$ & 0 & 0 & 1 & 1 & 0 & 1 \\
\hline \hline
\end{tabular}


for the positive parity channels, the three-quark operators defined in Tables VI, VII, and X of Ref. [17]. These compose a complete set of quasilocal operators plus the simplest set of nonlocal operators having one quark displaced relative to the other two.

The level ordering of baryon masses has been modeled using the spin-flavor SU(6) quark model. Negative parity multiplets are degenerate under SU(6) symmetry but the symmetry is broken by addition of a spin-spin contact interaction between quarks [19]. As a result, the lowest $N\left(J^{P}=\frac{1}{2}^{-}\right)$and $N\left(\frac{3}{2}^{-}\right)$states become less massive than the unperturbed levels and other states, such as $N\left(\frac{5}{2}^{-}\right),\left(\Delta_{2^{-}}^{-}\right)$, and $\Delta\left(\frac{3}{2}\right)$, become more massive. This pattern is observed in our lattice calculations. Degeneracies of $\mathrm{SU}(6)$ are further broken by addition of a spin-spin tensor interaction. In the positive parity excited states, Isgur and Karl [20] introduced an anharmonic perturbation in order to explain the mass splittings, and a hyperfine interaction was introduced to break the degeneracy of the multiplets. They found that the second excited $N\left(\frac{1}{2}^{+}\right)$state, the lowest $N\left(\frac{3}{2}^{+}\right)$state and the $N\left(\frac{5}{2}^{+}\right)$states are nearly degenerate. Our lattice results using a relatively large pion mass equal to $490 \mathrm{MeV}$ follow this pattern.

This paper is organized as follows. In Sec. II, we review the baryon operator construction and show the types of operators used in our simulations. In Sec. III the use of charge conjugation together with time reversal is discussed. The combination provides a means to obtain a second correlation matrix for each gauge configuration. In Sec. IV we review the computational techniques with special emphasis on the variational method that is essential for obtaining excited states. Also we discuss the anisotropic action that is employed and the tuning of parameters required to obtain the correct speed of light. Section V presents and discusses the results for energies and eigenvectors in each irrep and their stability with respect to the number of operators used in the variational method. Section VI discusses the pattern of lowest energy levels in the lattice results and shows that it is consistent with the physical spectrum. Two notable findings are (1) the first positive-parity excited state in the $I=\frac{1}{2}, G_{1 g}$ channel is found to have energy significantly higher than the experimentally known mass of the Roper resonance, and (2) spin assignments of either $\frac{5}{2}$ or $\frac{7}{2}$ are possible for some lattice levels that preferably would be assigned spin $\frac{5}{2}$ in order to match the pattern of the physical levels. Section VII gives a summary of the results.

\section{IMPROVED BARYON OPERATORS}

\section{A. Quasilocal operators}

We employ operators that transform as irreducible representations of the octahedral group. They are formed as linear combinations of elemental operators that have definite isospin and strangeness as follows,

$$
\bar{B}_{k}^{(\Lambda \lambda)}(\mathbf{x}, t)=c_{\alpha \beta \gamma, 0}^{(\Lambda \lambda k)} \bar{B}_{\alpha \beta \gamma}(\mathbf{x}, t),
$$

where $\alpha, \beta, \gamma$ are Dirac indices and the Clebsch-Gordan coefficients for the octahedral group provide the appropriate coefficients $c_{\alpha \beta \gamma, 0}^{(\Lambda \lambda k)}$. [17] The subscript " 0 " denotes quasilocal operators in which all quark fields have the same coordinates, $(\mathbf{x}, t)$. Octahedral group irreps are denoted by $\Lambda=\left\{G_{1 g}, G_{2 g}, H_{g}, G_{1 u}, G_{2 u}, H_{u}\right\}$ and the corresponding irrep dimensions are $d_{\Lambda}=\{2,2,4,2,2,4\}$, respectively. Each baryon operator carries a row label that distinguishes between the $d_{\Lambda}$ members of irrep $\Lambda$, i.e., $\lambda=1,2, \ldots, d_{\Lambda}$. When group representations contain $m_{\Lambda}$ occurrences of irrep $\Lambda$, the label $k$ is used to denote the $k$ th embedding.

Quasilocal elemental operators $\bar{B}_{\alpha \beta \gamma}$ in Eq. (1) are color singlet combinations of products of three quark fields, each of which is smeared in the same gauge-covariant fashion about point $\mathbf{x}$. They are listed in Table II for the different baryons. Although not shown in the table, each quark field has a color index $i, j$ or $k$, and a factor $\epsilon_{i j k}$ is included in order to construct a color-singlet combination. The Dirac index symmetries that give nonvanishing operators and the number of available quasilocal operators distributed over gerade irreps are also shown in the table.

Note that the "barred" form of the operator is given and it is formed from three barred quark fields. The "unbarred" form of baryon operator is obtained by a similar sum using complex conjugates of the same coefficients and three quark fields,

$$
B_{k}^{(\Lambda \lambda)}(\mathbf{x}, t)=c_{\alpha \beta \gamma, 0}^{(\Lambda \lambda k) *} B_{\alpha \beta \gamma}(\mathbf{x}, t) .
$$

TABLE II. Baryons and the corresponding quasilocal threequark elemental operators. Columns 1-4 show the symbol, isospin, strangeness, and the form of elemental quasilocal source operators with maximum $I_{z}$, respectively. In the next column, the MA label (mixed-antisymmetric) denotes combinations of three Dirac indices that are antisymmetric under permutation of the first two labels and orthogonal to the totally antisymmetric combination. Similarly, the MS label (mixed-symmetric) denotes combinations of indices that are symmetric with respect to the first two labels and orthogonal to the totally symmetric combination. Columns 6-8 show numbers of embeddings of operators with irreps $G_{1 g}, G_{2 g}$ and $H_{g}$, respectively. The last row shows the total number of even-parity operators, including all rows. Odd-parity operators are obtained from the even-parity ones as explained in the text.

\begin{tabular}{ccccccccc}
\hline \hline $\bar{B}$ & $I$ & $S$ & $\bar{B}_{\alpha \beta \gamma}$ & Dirac & $G_{1 g}$ & $G_{2 g}$ & $H_{g}$ & Total \\
\hline $\bar{N}$ & $1 / 2$ & 0 & $\left(\bar{u}_{\alpha} \bar{d}_{\beta}-\bar{d}_{\alpha} \bar{u}_{\beta}\right)_{\gamma} / \sqrt{2}$ & MA & 3 & 0 & 1 & 10 \\
$\bar{\Delta}$ & $3 / 2$ & 0 & $\bar{u}_{\alpha} \bar{u}_{\beta} \bar{u}_{\gamma}$ & S & 1 & 0 & 2 & 10 \\
$\bar{\Lambda}$ & 0 & -1 & $\left(\bar{u}_{\alpha} \bar{d}_{\beta}-\bar{d}_{\alpha} \bar{u}_{\beta}\right) \bar{s}_{\gamma} / \sqrt{2}$ & MA,A & 4 & 0 & 1 & 12 \\
$\bar{\Sigma}$ & 1 & -1 & $\bar{u}_{\alpha} \bar{u}_{\beta} \bar{s}_{\gamma}$ & MS,S & 4 & 0 & 3 & 20 \\
$\bar{\Xi}$ & $1 / 2$ & -2 & $\bar{s}_{\alpha} \bar{s}_{\beta} \bar{u}_{\gamma}$ & MS,S & 4 & 0 & 3 & 20 \\
$\bar{\Omega}$ & 0 & 3 & $\bar{s}_{\alpha} \bar{s}_{\beta} \bar{s}_{\gamma}$ & S & 1 & 0 & 2 & 10 \\
\hline \hline
\end{tabular}


Note that no $G_{2}$ irreps are obtained from quasilocal interpolating fields.

A quark field operator and a barred field operator are transformed into one another by charge conjugation as follows,

$$
\mathcal{C} \bar{q}_{\alpha} \mathcal{C}^{\dagger}=-C_{\alpha \alpha^{\prime}} q_{\alpha^{\prime}}, \quad \mathcal{C} q_{\alpha} \mathcal{C}^{\dagger}=\bar{q}_{\alpha^{\prime}} C_{\alpha^{\prime} \alpha^{\prime}}^{\dagger}
$$

where $C \equiv \gamma_{4} \gamma_{2}$. A three-quark operator that transforms according to irrep $\Lambda$ of the octahedral group is related by charge conjugation to an operator that transforms according to irrep $\Lambda_{c}$, which has opposite parity, i.e.,

$$
\begin{aligned}
\mathcal{C} \bar{B}_{k}^{(\Lambda \lambda)} \mathcal{C}^{\dagger} & =c_{\alpha \beta \gamma, 0}^{(\Lambda \lambda k)} \mathcal{C} \bar{B}_{\alpha \beta \gamma} \mathcal{C}^{\dagger}=-c_{\alpha \beta \gamma, 0}^{(\Lambda \lambda k)} \mathbf{C}_{\alpha \beta \gamma \alpha^{\prime} \beta^{\prime} \gamma^{\prime}} B_{\alpha^{\prime} \beta^{\prime} \gamma^{\prime}} \\
& \equiv-c_{\alpha^{\prime} \beta^{\prime} \gamma^{\prime}, 0}^{\left(\Lambda_{c} \lambda_{c} k\right)} B_{\alpha^{\prime} \beta^{\prime} \gamma^{\prime}}, \\
\mathcal{C} B_{k}^{(\Lambda \lambda)} \mathcal{C}^{\dagger} & =c_{\alpha \beta \gamma, 0}^{(\Lambda \lambda k) *} \mathcal{C} B_{\alpha \beta \gamma} \mathcal{C}^{\dagger}=c_{\alpha \beta \gamma, 0}^{(\Lambda \lambda k) *} \bar{B}_{\alpha^{\prime} \beta^{\prime} \gamma^{\prime}} \mathbf{C}_{\alpha^{\prime} \beta^{\prime} \gamma^{\prime} \alpha \beta \gamma}^{\dagger} \\
& =c_{\alpha^{\prime} \beta^{\prime} \gamma^{\prime}, 0}^{\left(\Lambda_{c} \lambda_{k} k\right) *} \bar{B}_{\alpha^{\prime} \beta^{\prime} \gamma^{\prime}},
\end{aligned}
$$

where $\mathbf{C}_{\alpha^{\prime} \beta^{\prime} \gamma^{\prime} \alpha \beta \gamma}=C_{\alpha^{\prime} \alpha} C_{\beta^{\prime} \beta} C_{\gamma^{\prime} \gamma}$. If $\Lambda$ is $G_{1 g}, G_{2 g}$ or $H_{g}$, then $\Lambda_{c}$ is $G_{1 u}, G_{2 u}$ or $H_{u}$, respectively, and $\lambda_{c}=d_{\Lambda}+$ $1-\lambda$. This one-to-one correspondence between an operator of one parity and an operator with the opposite parity holds for any quasilocal or displaced operator. Choosing the gerade operators according to Eq. (1) and the ungerade operators according to the right side of Eq. (4) provides a convenient labeling of operators that facilitates the use of charge-conjugation relations to realize improved statistics, as explained in Sec. III.

\section{B. One-link-displaced operators}

A simple extension of quasilocal operators involves displacement of the third quark field along a spatial direction relative to the first two quark fields, with a gauge link included in order to maintain gauge covariance. An abbreviated notation for displacements uses the operator $\hat{d}_{\ell}$ whose action on a three-quark quasilocal operator is defined as follows,

$$
\begin{aligned}
\hat{d}_{\ell} \bar{B}_{k}^{(\Lambda \lambda)}(\mathbf{x}, t)= & \epsilon_{i j k} f_{a b c}^{(I S)} c_{\alpha \beta \gamma}^{(\Lambda \lambda k)} \overline{\tilde{q}}_{\alpha}^{i, a}(\mathbf{x}, t) \overline{\tilde{q}}_{\beta}^{i, b}(\mathbf{x}, t) \\
& \times \overline{\tilde{q}}_{\gamma}^{k^{\prime}, c a}(\mathbf{x}+\hat{\ell}, t) U_{\ell}^{\dagger \ell^{\prime} k}(\mathbf{x}, t),
\end{aligned}
$$

where $\hat{\ell}$ can take any of six spatial directions, $\pm x, \pm y, \pm z$.

Displacement operators transform amongst themselves under lattice rotations. Linear combinations of displacements provide suitable bases for the construction of irreps. We define six linearly independent combinations of displacements and the operators that create them as follows,

$$
\left(\begin{array}{llllll}
\hat{A}_{1} \bar{B} & \hat{D}_{+} \bar{B} & \hat{D}_{-} \bar{B} & \hat{D}_{0} \bar{B} & \hat{E}_{0} \bar{B} & \hat{E}_{2} \bar{B}
\end{array}\right)^{T} \equiv\left(\begin{array}{c}
\frac{1}{\sqrt{6}}\left(\hat{d}_{x} \bar{B}+\hat{d}_{y} \bar{B}+\hat{d}_{z} \bar{B}+\hat{d}_{-x} \bar{B}+\hat{d}_{-y} \bar{B}+\hat{d}_{-z} \bar{B}\right) \\
\frac{i}{2}\left[\left(\hat{d}_{x} \bar{B}-\hat{d}_{-x} \bar{B}\right)+i\left(\hat{d}_{y} \bar{B}-\hat{d}_{-y} \bar{B}\right)\right] \\
-\frac{i}{2}\left[\left(\hat{d}_{x} \bar{B}-\hat{d}_{-x} \bar{B}\right)-i\left(\hat{d}_{y} \bar{B}-\hat{d}_{-y} \bar{B}\right)\right] \\
-\frac{i}{\sqrt{2}}\left(\hat{d}_{z} \bar{B}-\hat{d}_{-z} \bar{B}\right) \\
\frac{1}{\sqrt{12}}\left[2\left(\hat{d}_{z} \bar{B}+\hat{d}_{-z} \bar{B}\right)-\left(\hat{d}_{x} \bar{B}+\hat{d}_{-x} \bar{B}\right)-\left(\hat{d}_{y} \bar{B}+\hat{d}_{-y} \bar{B}\right)\right] \\
\frac{1}{2}\left[\left(\hat{d}_{x} \bar{B}+\hat{d}_{-x} \bar{B}\right)-\left(\hat{d}_{y} \bar{B}+\hat{d}_{-y} \bar{B}\right)\right]
\end{array}\right)
$$

where displacement operators denoted as $\hat{A}_{1}, \hat{D}_{ \pm, 0}$, and $\hat{E}_{0,2}$ create combinations of operators that transform according to the $A_{1}, T_{1}$, and $E$ single-valued irreps of the octahedral group, respectively. As may be seen in Table III, the lowest angular momentum in $A_{1}$ is $L=0$, the lowest in $T_{1}$ is $L=1$, and the lowest in $E$ is $L=2$. The combinations of displacements are chosen so that they transform as the lattice discretizations of the spherical harmonics $Y_{L m}$, i.e., $\hat{A}_{1} \sim Y_{00}, \hat{D}_{+, 0,-} \sim Y_{11}, Y_{10}, Y_{1-1}$, and $\hat{E}_{0,2} \sim Y_{20}$, $\left(Y_{22}+Y_{2-2}\right)$. Using this convention, the $A_{1}, T_{1}$, and $E$ one-link-displaced operators are defined as

$$
\begin{aligned}
\bar{B}_{k}^{(\Lambda \lambda)} & =\left\{\begin{array}{l}
\hat{A}_{1} \bar{B}_{k^{\prime}}^{(\Lambda \lambda)} \\
\sum_{r, \lambda^{\prime}} C\left(\begin{array}{ccc}
\Lambda & T_{1} & \Lambda^{\prime} \\
\lambda & r & \lambda^{\prime}
\end{array}\right) \hat{D}_{r} \bar{B}_{k^{\prime}}^{\left(\Lambda^{\prime} \lambda^{\prime}\right)} \\
\sum_{r, \lambda^{\prime}} C\left(\begin{array}{ccc}
\Lambda & E & \Lambda^{\prime} \\
\lambda & r & \lambda^{\prime}
\end{array}\right) \hat{E}_{r} \bar{B}_{k^{\prime}}^{\left(\Lambda^{\prime} \lambda^{\prime}\right)}
\end{array}\right. \\
& \equiv c_{\alpha \beta \gamma, \ell}^{(\Lambda \lambda k)} \hat{d}_{\ell} \bar{B}_{\alpha \beta \gamma}
\end{aligned}
$$

respectively, where

$$
C\left(\begin{array}{ccc}
\Lambda & \Lambda^{\prime} & \Lambda^{\prime \prime} \\
\lambda & \lambda^{\prime} & \lambda^{\prime \prime}
\end{array}\right)
$$

are Clebsch-Gordan coefficients appropriate for forming overall irrep $\Lambda$ from direct products of irreps $\Lambda^{\prime}$ and $\Lambda^{\prime \prime}$. The coefficients follow the conventions of Ref. [17]. The second line of Eq. (7) defines new coefficients for linear

TABLE III. The number of occurrences of single-valued irrep $\Lambda$ of the octahedral group for integer values of continuum angular momentum $L$.

\begin{tabular}{lccccc}
\hline \hline$\Lambda$ & $\mathrm{L}=0$ & 1 & 2 & 3 & 4 \\
\hline$A_{1}$ & 1 & 0 & 0 & 0 & 1 \\
$T_{1}$ & 0 & 1 & 0 & 1 & 1 \\
$E$ & 0 & 0 & 1 & 0 & 1 \\
$T_{2}$ & 0 & 0 & 1 & 1 & 1 \\
$A_{2}$ & 0 & 0 & 0 & 1 & 0 \\
\hline \hline
\end{tabular}


TABLE IV. Numbers of embeddings of even-parity irreps, $G_{1 g}, G_{2 g}$, and $H_{g}$, that are obtained with one-link-displaced operators for different baryons. Columns show the number of operators for each overall irrep that can be made using the three irreps of one-link displacements: $A_{1}, T_{1}$, and $E$. Operators corresponding to a total derivative acting on a three-quark operator are omitted because they vanish when projected to zero momentum.

\begin{tabular}{lcccr}
\hline \hline $\bar{B}$ & Disp. & $G_{1 g}$ & $G_{2 g}$ & $H_{g}$ \\
\hline \multirow{N}{N}{} & $A_{1}$ & 8 & 0 & 4 \\
& $T_{1}$ & 8 & 3 & 11 \\
$\bar{\Delta}, \bar{\Omega}^{-}$ & $E$ & 4 & 4 & 12 \\
& $A_{1}$ & 4 & 0 & 3 \\
& $T_{1}$ & 4 & 1 & 5 \\
$\bar{\Lambda}$ & $E$ & 3 & 3 & 7 \\
& $A_{1}$ & 4 & 0 & 1 \\
& $T_{1}$ & 5 & 1 & 6 \\
$\bar{\Sigma}, \bar{\Xi}$ & $E$ & 1 & 1 & 5 \\
& $A_{1}$ & 4 & 0 & 3 \\
& $T_{1}$ & 7 & 3 & 10 \\
\hline \hline
\end{tabular}

combinations of displaced three-quark operators that realize the $A_{1}, T_{1}$, or $E$-type operators of Eq. (6). Table IV shows the numbers of positive-parity, one-link-displaced operators for different baryons. For the one-link-displaced operators, there are inequivalent operators for each of the two ways that $I=\frac{1}{2}$ can be formed from three light quarks, namely, the mixed-antisymmetric (MA) and mixedsymmetric (MS) combinations. Operators corresponding to total derivatives are excluded because they vanish when projected to zero total momentum.

We construct matrices of correlation functions

$$
\begin{aligned}
C_{k k^{\prime}}^{(\Lambda \lambda)}(t) \delta_{\Lambda \Lambda^{\prime}} \delta_{\lambda \lambda^{\prime}}= & c_{\alpha \beta \gamma, \ell}^{(\Lambda \lambda k) *} c_{\mu \nu \rho, \ell^{\prime}}^{\left(\Lambda^{\prime} \lambda^{\prime} k^{\prime}\right)} \sum_{\mathbf{x}}\langle 0| B_{\alpha \beta \gamma}(\mathbf{x}, t) \hat{d}_{\ell}^{\dagger} \hat{d}_{\ell^{\prime}} \\
& \times \bar{B}_{\alpha^{\prime} \beta^{\prime} \gamma^{\prime}}(\mathbf{0}, 0)|0\rangle \gamma_{\alpha^{\prime} \mu}^{4} \gamma_{\beta^{\prime} \nu}^{4} \gamma_{\gamma^{\prime} \rho}^{4}
\end{aligned}
$$

where displacement operator $\hat{d}_{\ell}$ applied to baryon fields denotes a one-link-displaced baryon field for $\ell=$ $\pm x, \pm y, \pm z$ and a quasilocal baryon for $\ell=0$. Lattice operators belonging to different irreps or different rows are orthogonal because of the octahedral symmetry of the lattice. Different embeddings of a given irrep and row provide sets of operators that we used in calculating the matrix of correlation functions. The $\gamma_{4}$ matrices are included in Eq. (8) in order to produce a Hermitian matrix of correlation functions. For the operators used in this work, the $\gamma_{4}$ matrices always reduce to a factor $\mathcal{P}_{k^{\prime}}^{(\Lambda)}= \pm 1$ when they act on the three barred quark fields in $\bar{B}_{k}^{(\Lambda \lambda)}$. This factor is the $\rho$ parity defined in Ref. [17]. We write a matrix of correlation functions for irrep $\Lambda$ and row $\lambda$ as

$$
C_{k k^{\prime}}^{(\Lambda \lambda)}(t)=\mathcal{P}_{k^{\prime}}^{(\Lambda)} \sum_{\mathbf{x}}\left\langle 0\left|B_{k}^{(\Lambda \lambda)}(\mathbf{x}, t) \bar{B}_{k^{\prime}}^{(\Lambda \lambda)}(\mathbf{0}, 0)\right| 0\right\rangle .
$$

\section{CHARGE CONJUGATION}

By inserting complete sets of particle states $|n\rangle$ and antiparticle states $|\bar{n}\rangle$ in Eq. (9), and using translational invariance to extract the dependence on $\mathbf{x}$ and $t$, correlation functions are expanded as

$$
\begin{aligned}
C_{k k^{\prime}}^{(\Lambda \lambda)}(t)= & \mathcal{P}_{k^{\prime}} \delta_{\mathbf{P}, 0}\left[\sum_{n} \theta(t)\left\langle 0\left|B_{k}^{(\Lambda \lambda)}\right| n\right\rangle\left\langle n\left|\bar{B}_{k^{\prime}}^{(\Lambda \lambda)}\right| 0\right\rangle e^{-E_{n} t}\right. \\
& \left.-\sum_{\bar{n}} \theta(-t)\left\langle 0\left|\bar{B}_{k^{\prime}}^{(\Lambda \lambda)}\right| \bar{n}\right\rangle\left\langle\bar{n}\left|B_{k}^{(\Lambda \lambda)}\right| 0\right\rangle e^{E_{\bar{n}} t}\right] .
\end{aligned}
$$

Using the charge conjugation relations $|\bar{n}\rangle=\mathcal{C}|n\rangle e^{i \phi}$, and the invariance of the vacuum state under charge conjugation, the antiparticle contributions in the $t<0$ part of Eq. (10) are rewritten as

$$
\begin{aligned}
\mathcal{P}_{k^{\prime}}^{(\Lambda)} & \left\langle 0\left|\mathcal{C} \bar{B}_{k^{\prime}}^{(\Lambda \lambda)} \mathcal{C}^{\dagger} \mathcal{C}\right| \bar{n}\right\rangle\left\langle\bar{n}\left|\mathcal{C}^{\dagger} \mathcal{C} B_{k}^{(\Lambda \lambda)} \mathcal{C}^{\dagger}\right| 0\right\rangle \\
= & \mathcal{P}_{k^{\prime}}^{\left(\Lambda_{c}\right)} c_{\alpha \beta \gamma, \ell}^{\left(\Lambda_{c} \lambda_{c} k\right) *} c_{\alpha^{\prime} \beta^{\prime} \gamma^{\prime}, \ell^{\prime}}^{\left(\Lambda_{c} \lambda_{c} k^{\prime}\right)}\left\langle 0\left|B_{\alpha \beta \gamma} \hat{d}_{\ell}^{\dagger}\right| n\right\rangle^{*} \\
& \times\left\langle n\left|\hat{d}_{\ell^{\prime}} \bar{B}_{\alpha^{\prime} \beta^{\prime} \gamma^{\prime}}\right| 0\right\rangle^{*},
\end{aligned}
$$

where $\mathcal{P}_{k^{\prime}}^{\left(\Lambda_{c}\right)}=-\mathcal{P}_{k^{\prime}}^{(\Lambda)}$. The relation between correlation functions with different parities is then [18]

$$
C_{k k^{\prime}}^{(\Lambda \lambda)}(t)=-C_{k k^{\prime}}^{\left(\Lambda_{c} \lambda_{c}\right) *}(-t) \text {. }
$$

Applying the temporal lattice boundary conditions $C_{k k^{\prime}}^{(\Lambda \lambda)}(-t)=\eta_{t} C_{k k^{\prime}}^{(\Lambda \lambda)}(T-t)$, with $\eta_{t}=+1$ for periodic boundary conditions and $\eta_{t}=-1$ for antiperiodic boundary conditions, the matrix of correlation functions can be written as

$$
\begin{aligned}
C_{k k^{\prime}}^{(\Lambda \lambda)}(t)= & \delta_{\mathbf{P}, 0} \sum_{n}\left[\theta(t) \mathcal{P}_{k^{\prime}}^{(\Lambda)}\left\langle 0\left|B_{k}^{(\Lambda \lambda)}\right| n\right\rangle\left\langle n\left|\bar{B}_{k^{\prime}}^{(\Lambda \lambda)}\right| 0\right\rangle e^{-E_{n} t}\right. \\
& -\eta_{t} \theta(T-t) \mathcal{P}_{k^{\prime}}^{\left(\Lambda_{c}\right)}\left\langle 0\left|B_{k}^{\left(\Lambda_{c} \lambda_{c}\right)}\right| n\right\rangle^{*} \\
& \left.\times\left\langle n\left|\bar{B}_{k^{\prime}}^{\left(\Lambda_{c} \lambda_{c}\right)}\right| 0\right\rangle^{*} e^{-E_{\bar{n}}(T-t)}\right]
\end{aligned}
$$

in the interval $0 \leq t<T$. The forward propagating signal of a correlation function is equal to the backward propagating signal of the parity-reversed, complex-conjugated correlation function within the factor $-\eta_{t}$, i.e.,

$$
C_{k k^{\prime}}^{(\Lambda \lambda)}(t)=-\eta_{t} C_{k k^{\prime}}^{\left(\Lambda_{c} \lambda_{c}\right) *}(T-t) .
$$

This symmetry is used to improve statistics. For each gauge configuration we compute matrices of correlation functions using for positive-parity operators the $\bar{B}^{(\Lambda)}$-type operators and for the corresponding negative-parity operators the $\bar{B}^{\left(\Lambda_{c}\right)}$-type operators. Starting with a matrix of correlation functions for a given parity, a second matrix of correlation functions is obtained for the opposite parity. After time-reversal, complex conjugation and multiplica- 
tion by the factor $-\eta_{t}$, the second matrix of correlation functions is averaged with the first one. A typical state created by the $\bar{B}_{k}^{(\Lambda \lambda)}$ operator has an effective mass plateau occurring in first half of the time extent, $t<T / 2$. Using the $\bar{B}_{k}^{\left(\Lambda_{c} \lambda_{c}\right)}$ operator, a similar plateau occurs at $t>T / 2$. These two plateaus are largely statistically independent samples because gauge configurations on time slices significantly before $T / 2$ are largely uncorrelated with ones on time slices significantly after $T / 2$.

\section{COMPUTATIONAL TECHNIQUES}

\section{A. Variational method}

Analysis of excited state energies is based upon the matrices of correlation functions defined in Eq. (9). Statistics are improved by averaging over the rows of irreps, all of which are equivalent because of the cubic symmetry. The matrix averaged over rows is denoted as $C_{k k^{\prime}}^{(\Lambda)}(t)$, i.e., the row label is omitted.

Because the operators used to form a matrix of correlation functions are not normalized with respect to one another, we find that they can produce diagonal elements of a correlation matrix that differ by 2 orders of magnitude. It is convenient to adopt a normalization scheme at time $t_{0}$ such that each operator produces a diagonal matrix element equal to 1 . Thus, we define normalization factors,

$$
N_{k}\left(t_{0}\right)=\frac{1}{\sqrt{\left|C_{k k}^{(\Lambda)}\left(t_{0}\right)\right|}},
$$

and renormalize the operators by attaching a factor $N_{k}$ to operator $B_{k}$. The new matrix of correlation functions is

$$
\tilde{C}_{k k^{\prime}}^{(\Lambda)}(t)=N_{k} C_{k k^{\prime}}^{(\Lambda)}(t) N_{k^{\prime}},
$$

and its diagonal elements obey $\tilde{C}_{k k}^{(\Lambda)}\left(t_{0}\right)=1$. Because of this normalization convention the components of eigenvectors indicate the relative importance of the contributions of various operators to an eigenstate.

In order to extract an energy spectrum from the matrix of correlation functions, we numerically solve the following generalized eigenvalue equation,

$$
\sum_{k^{\prime}} \tilde{C}_{k k^{\prime}}^{(\Lambda)}(t) v_{k^{\prime}}^{(n)}\left(t, t_{0}\right)=\alpha^{(n)}\left(t, t_{0}\right) \sum_{k^{\prime}} \tilde{C}_{k k^{\prime}}^{(\Lambda)}\left(t_{0}\right) v_{k^{\prime}}^{(n)}\left(t, t_{0}\right),
$$

where superscript $n$ labels the eigenstates, such as the ground state, the first excited state, and so forth. The reference time $t_{0}$ in Eq. (16) is taken near the source time $t=0$ in order to have significant contributions from excited states and to ensure stability of the Cholesky procedure below. The principal eigenvalues $\alpha^{(n)}\left(t, t_{0}\right)$ are related to the energy $E_{n}$ by [16]

$$
\alpha^{(n)}\left(t, t_{0}\right) \simeq e^{-E_{n}\left(t-t_{0}\right)}\left(1+\mathcal{O}\left(e^{-|\delta E| t}\right)\right),
$$

where $\delta E$ is the difference between $E_{n}$ and the next closest energy.

Energies $E_{n}$ are calculated from the principal eigenvalues according to

$$
E_{n}=-\ln \left[\frac{\alpha^{(n)}\left(t+1, t_{0}\right)}{\alpha^{(n)}\left(t, t_{0}\right)}\right] .
$$

We also perform fits of $\alpha^{(n)}\left(t, t_{0}\right)$ to an exponential function $\alpha^{(n)}\left(t, t_{0}\right)=e^{-E_{n}\left(t-t_{0}\right)}$ over a range of time slices in order to better determine the error of the energy $E_{n}$.

Numerical solutions of the generalized eigenvalue equation are obtained by performing a Cholesky decomposition of the matrix of correlation functions at the reference time, $t_{0}$,

$$
\tilde{C}_{k k^{\prime}}^{(\Lambda)}\left(t_{0}\right)=A_{k k^{\prime \prime}}^{T} A_{k^{\prime \prime} k^{\prime}}
$$

For the transfer matrix,

$$
T\left(t, t_{0}\right)=\left(A^{T}\right)^{-1} \tilde{C}^{(\Lambda)}(t) A^{-1},
$$

the eigenvalue problem is

$$
\begin{gathered}
T_{k k^{\prime}}\left(t, t_{0}\right) V_{k^{\prime}}^{(n)}\left(t, t_{0}\right)=\alpha^{(n)}\left(t, t_{0}\right) V_{k}^{(n)}\left(t, t_{0}\right), \\
V_{k}^{(n)}\left(t, t_{0}\right)=A_{k k^{\prime}} v_{k^{\prime}}^{(n)}\left(t, t_{0}\right) .
\end{gathered}
$$

Left and right eigenvectors of the transfer matrix $T_{k k^{\prime}}\left(t, t_{0}\right)$ are the same.

Eigenvectors in the generalized eigenvalue equation, Eq. (16), are orthogonal with respect to $\tilde{C}_{k k^{\prime}}^{(\Lambda)}\left(t_{0}\right)$, i.e.,

$$
v_{k}^{(n) T}\left(t, t_{0}\right) \tilde{C}_{k k^{\prime}}^{(\Lambda)}\left(t_{0}\right) v_{k^{\prime}}^{\left(n^{\prime}\right)}\left(t, t_{0}\right)=\delta_{n n^{\prime}} .
$$

It follows from Eqs. (16) and (23) that the correlation matrix at time $t$ is diagonalized by these vectors, i.e.,

$$
\boldsymbol{v}_{k}^{(n) T}\left(t, t_{0}\right) \tilde{C}_{k k^{\prime}}^{(\Lambda)}(t) v_{k^{\prime}}^{\left(n^{\prime}\right)}\left(t, t_{0}\right)=\alpha^{(n)}\left(t, t_{0}\right) \delta_{n n^{\prime}} .
$$

It is useful to define improved operators by first adopting a suitable normalization of the eigenvector components. Let

$$
\tilde{\boldsymbol{v}}_{k}^{(n)}\left(t, t_{0}\right) \equiv Z_{n}(t) N_{k} \boldsymbol{v}_{k}^{(n)}\left(t, t_{0}\right),
$$

such that

$$
\sum_{k}\left|\tilde{\boldsymbol{v}}_{k}^{(n)}\left(t, t_{0}\right)\right|^{2}=1 .
$$

The factor $Z_{n}$ allows a comparison of the overall normalization of eigenvectors calculated on different volumes.

\section{B. Lattice setup and tuning}

A technical difficulty in extracting excited state energies from lattice QCD simulations is that the signal-to-noise ratio degrades for increasing time. A plateau in the energy may not be realized for excitations. We use several techniques to overcome this problem, namely, the optimization of operators so as to achieve early plateaus of the energies, 
the imposition of symmetries and the use of more gauge configurations to increase the statistics, and the use of anisotropic lattices. Anisotropic lattices are designed with temporal lattice spacing $a_{t}$ smaller than spatial lattice spacing $a_{s}$. Using a finer lattice spacing along the time direction provides more time slices for analysis of correlation functions at small time separations from the source. Of course anisotropic lattices require tuning of coefficients in the action.

In this work, anisotropic lattices with two different volumes are used: 239 gauge field configurations on a $16^{3} \times 64$ lattice and 167 configurations on a $24^{3} \times 64$ lattice. For both lattices, the renormalized ratio of spatial lattice spacing to temporal spacing is $\xi=a_{s} / a_{t}=3.0$ and the temporal lattice spacing corresponds to $a_{t}^{-1}=$ $6.0 \mathrm{GeV}$ [8]. Gauge-field configurations are generated using the anisotropic, unimproved Wilson gauge action in the quenched approximation with $\beta=6.1$

\section{Anisotropic Wilson gauge action}

The anisotropic Wilson gauge action [21-24] is given by

$$
\begin{aligned}
S_{G}^{\xi}= & \frac{\beta}{N_{c}}\left[\frac{1}{\xi_{0}} \sum_{x, s>s^{\prime}} \operatorname{Re} \operatorname{Tr}\left(1-P_{s s^{\prime}}(x)\right)\right. \\
& \left.+\xi_{0} \sum_{x, s} \operatorname{Re} \operatorname{Tr}\left(1-P_{s t}(x)\right)\right] .
\end{aligned}
$$

The renormalized anisotropy $\xi=3$ is held fixed in our calculations and the bare anisotropy, $\xi_{0}$, is varied in order to obtain the target value of $\xi$ for the desired value of $\beta=$ 6.1. Because the quenched approximation is used, tuning of the gauge action may be performed independently of the fermion action. Determination of the renormalized anisotropy is based on measurements of static potentials for a quark-antiquark pair.

\section{Anisotropic Wilson fermion action}

The anisotropic Wilson fermion action has the form

$$
\begin{aligned}
S_{F}^{\xi}= & a_{t} a_{s}^{3} \sum_{x} \bar{q}(x)\left[m_{0}+\nu_{t}\left(\gamma_{4} \nabla_{t}-\frac{a_{t}}{2} \Delta_{t}\right)\right. \\
& \left.+\nu_{s} \sum_{s}\left(\gamma_{s} \nabla_{s}-\frac{a_{s}}{2} \Delta_{s}\right)\right] q(x),
\end{aligned}
$$

where

$$
\begin{aligned}
& \Delta_{\mu} q(x)=\frac{U_{\mu}(x) q(x+\hat{\mu})+U_{\mu}^{\dagger}(x-\hat{\mu}) q(x-\hat{\mu})-2 q(x)}{a_{\mu}^{2}}, \\
& \nabla_{\mu} q(x)=\frac{U_{\mu}(x) q(x+\hat{\mu})-U_{\mu}^{\dagger}(x-\hat{\mu}) q(x-\hat{\mu})}{2 a_{\mu}} .
\end{aligned}
$$

Note that when $\nu_{t}=\nu_{s}$, the fermion action in Eq. (28) becomes the original Wilson fermion action [25]. We hold $\nu_{t}=1$ in the tuning because that preserves the projection property of $\left(1 \pm \gamma_{4}\right) / 2$ for parity. Then $\nu_{s}$ and the bare

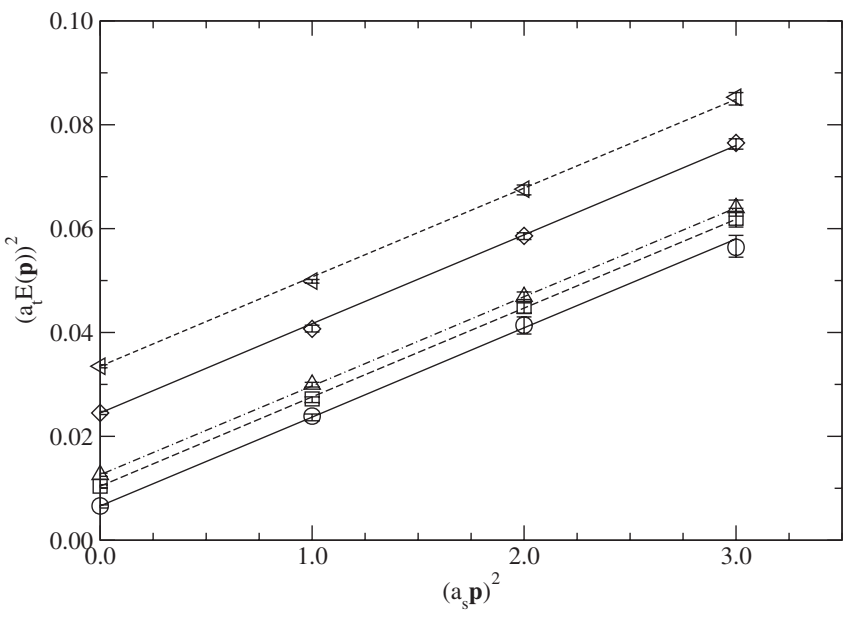

FIG. 1. Pion dispersion relation based on tuned parameters $m_{0}$ and $\nu_{s}$. The renormalized anisotropy is 3.0 and the lattice volume is $16^{3} \times 64$ with $\beta=6.1$. Lines show the $c=1$ dispersion relation. The meaning of symbols is given in Table V.

quark mass $m_{0}$ are tuned in order to obtain the desired pion mass and the correct speed of light.

The tuning is based on the relativistic dispersion relation for the pion, $E^{2}(\mathbf{p})=m^{2} c^{4}+c^{2} \mathbf{p}^{2}$, where $E(\mathbf{p})$ is the energy of a pion with total momentum $\mathbf{p}$ and $m c^{2}=$ $E(0)$. Expressing quantities in terms of the lattice spacing, the speed of light is

$$
c(\mathbf{p})=\xi \sqrt{\frac{\left(a_{t} E(\mathbf{p})\right)^{2}-\left(a_{t} E(0)\right)^{2}}{\left(a_{s} \mathbf{p}\right)^{2}}},
$$

where $\xi$ is the renormalized anisotropy. We measured the pion energy $E(\mathbf{p})$ for $\mathbf{p}=0$ and the lowest three nonzero values of momentum, namely, $\mathbf{p}=\frac{2 \pi}{L}(1,0,0), \frac{2 \pi}{L}(1,1,0)$, and $\frac{2 \pi}{L}(1,1,1)$. We tuned $\nu_{s}$ for five different bare quark masses $m_{0}$. The dispersion relation for tuned parameter $\nu_{s}$ on the $16^{3} \times 64$ lattice is plotted in Fig. 1. The straight lines passing through the mean value of $E^{2}(0)$ show the desired continuum dispersion relation for each pion mass. In physical units, the volume is about $(1.6 \mathrm{fm})^{3} \times$ (2.1 fm).

For the anisotropic, unimproved Wilson fermion action, tuning of the fermion action produced the results given in Table V. A periodic boundary condition is employed for

TABLE V. Tuning parameters for the fermion action on the $16^{3} \times 64$ lattice using renormalized anisotropy $\xi=3$ and $\nu_{t}=$ 1

\begin{tabular}{lcccc}
\hline \hline Symbol & $a_{t}^{2} m_{\pi}^{2}$ & $m_{\pi}(\mathrm{MeV})$ & $m_{0}$ & $\nu_{s}$ \\
\hline$\bigcirc$ & $0.0066(5)$ & 490 & -0.313 & 0.898 \\
$\square$ & $0.0104(5)$ & 610 & -0.318 & 0.901 \\
$\triangle$ & $0.0126(5)$ & 675 & -0.305 & 0.902 \\
$\diamond$ & $0.0224(5)$ & 900 & -0.290 & 0.910 \\
$\triangleleft$ & $0.0335(5)$ & 1100 & -0.280 & 0.915 \\
\hline \hline
\end{tabular}


spatial directions and an antiperiodic boundary condition is employed for the temporal direction. Our spectrum calculations are based on the parameter set that yields a pion mass of $490 \mathrm{MeV}$.

Gauge links are smeared at source and sink according to the APE-smearing method [12],

$U_{j}^{(n+1)}(x) \rightarrow U_{j}^{(n)}(x)+\frac{1}{\alpha} \sum_{k \perp j} U_{k}^{(n)}(x) U_{j}^{(n)}(x+\hat{k}) U_{k}^{\dagger(n)}(x+\hat{j})$,

where projection of gauge link matrices to $\mathrm{SU}(3)$ is performed after each iteration. We used the APE-smearing parameters $(\alpha, n)=(2.5,3)$ throughout this work.

Quark fields are smeared for both quasilocal and onelink-displaced operators using the Gaussian smearing method [13],

$$
q_{\mu}(x, t) \rightarrow \sum_{x^{\prime}} \hat{G}^{(N)}\left(x, x^{\prime}\right) q_{\mu}\left(x^{\prime}, t\right),
$$

where $\hat{G}^{(N)}$ is an operator that acts recursively,

$$
\begin{aligned}
& \hat{G}^{(N)}\left(x, x^{\prime}\right)=\sum_{y}\left(\delta_{x, y}+\sigma^{2} \nabla_{x, y}^{2} / 4 N\right) \hat{G}^{(N-1)}\left(y, x^{\prime}\right), \\
& \hat{G}^{(0)}\left(x, x^{\prime}\right)=\delta_{x, x^{\prime}},
\end{aligned}
$$

and $\nabla_{x, x^{\prime}}^{2}$ is a three-dimensional, gauge-covariant Laplacian operator. We used $(\sigma, N)=(3.0,20)$ in this calculation.

\section{DISCUSSION OF RESULTS FOR EACH SYMMETRY CHANNEL}

We have extracted energies for $I=\frac{1}{2}$ and $I=\frac{3}{2}$ channels by diagonalizing matrices of correlation functions formed from three-quark operators that share the same octahedral symmetry. A large number of operators is available so that reduction to a set of the most important operators is carried out in the initial stage. The available operators are divided into several subsets containing roughly 3 to 8 operators each and we diagonalize the matrices computed from each subset of operators. Inspection of the energies and corresponding eigenvectors reveals which operators provide stronger signals for the low-lying states of interest. In the second stage we omit all but these good operators and form new matrices of correlation functions, which are then diagonalized. This two-stage procedure yields solid results for energies of low-lying states and is less susceptible to noise than applying the variational method directly to the matrices of largest dimension.

In each symmetry channel, we have studied how the energies depend upon the number of good operators that are used in applying the variational method. Starting from a given set of good operators, matrices of correlation functions of reduced dimension are obtained by omitting a less important operator from the selected set. Continuing this process, we have diagonalized matrices of various dimensions and obtained energies. Low-lying energies in each symmetry channel are plotted as a function of matrix dimension in Fig. 2. The $I=\frac{3}{2}, G_{2 g / u}$ channel is not shown because results in that channel are based on a single
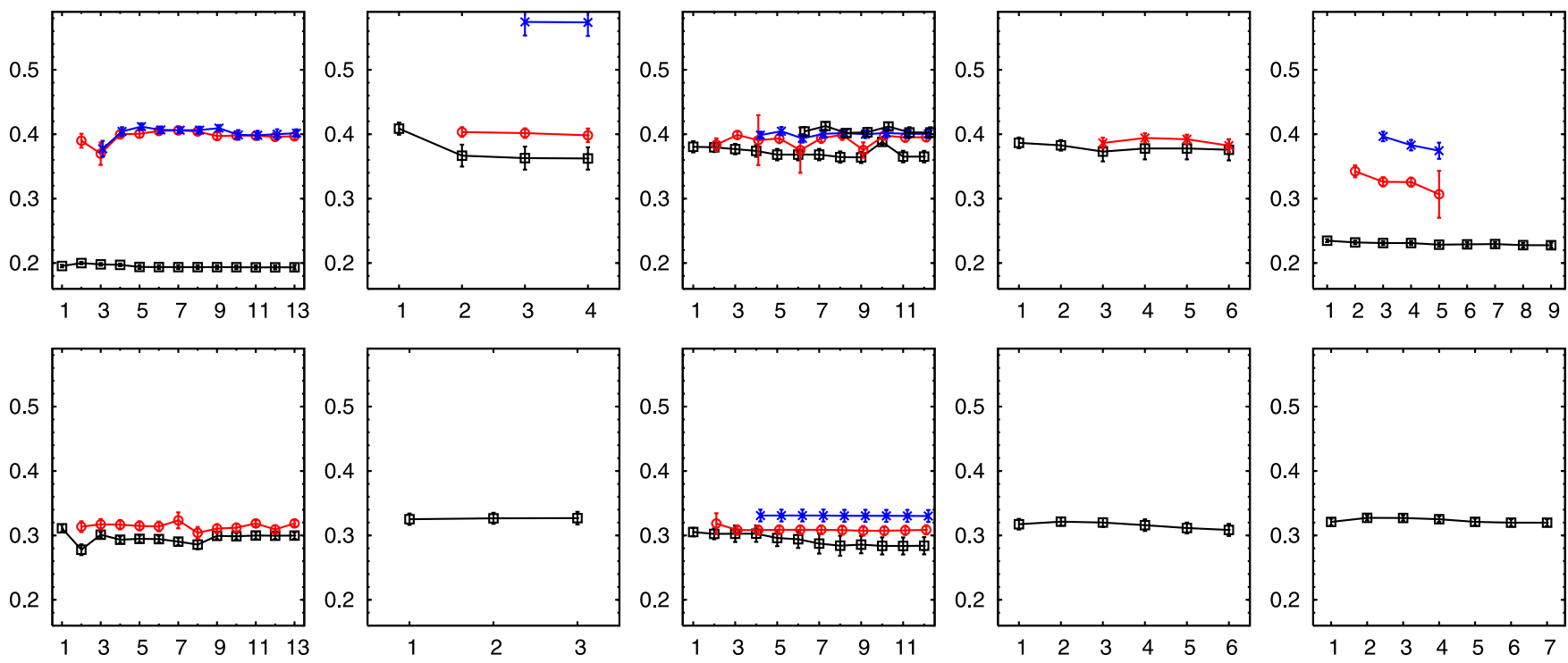

(a) $N, G_{1}$

(b) $N, G_{2}$

(c) $N, H$

(d) $\Delta, G_{1}$

(e) $\Delta, H$

FIG. 2 (color online). Energies in lattice units versus dimension of the matrices of correlation functions. Data for the $24^{3} \times 64$ lattice are used. Upper panels show energies for positive parity states and lower panels show energies for negative parity states. The $I=\frac{3}{2}$, $G_{2 g / u}$ states are excluded because correlation functions are based on a single operator. 
operator. The lowest energy states are more or less stable as long as a few important operators are retained. However, it is expected that use of more than the minimum number of operators improves the lowest-lying states because it reduces contamination from higher lying states. This expectation appears to hold true for the lowest and first excited states but is less evident for higher excited states with the limited set of operators used in this work.
Diagonalization of a matrix of correlation functions provides the principal eigenvalues $\alpha^{(n)}\left(t, t_{0}\right)$. These have been fit with a single exponential form over a selected range of times, $t_{1} \leq t \leq t_{2}$ in order to extract energies, i.e., $\alpha^{(n)}\left(t, t_{0}\right)=C e^{-E_{n}\left(t-t_{0}\right)}$. The resulting energies are given in Tables VI, VII, VIII, and IX, which show the energies obtained for positive-parity states with $I=\frac{1}{2}$, negative-parity states with $I=\frac{1}{2}$, positive-parity states

TABLE VI. The $I=\frac{1}{2}, N^{*}$, positive-parity energies in the units of $a_{t}^{-1}$ obtained on a $24^{3} \times 64$ lattice (left) and a $16^{3} \times 64$ lattice (right). The irrep is shown in the first row together with the dimension of the matrix of correlation functions used. In the first column, energy values obtained from fitting the principal eigenvalues are shown in increasing order, namely, the second row contains energies of the lowest-lying states for each channel and the third row contains the first excited state energies, etc. The Z-factor of Eq. (25) is given in the second column. A time range in which the energy and the Z-factor are fitted on the $24^{3} \times 64$ lattice is listed in the third column.

\begin{tabular}{ccc|cc}
\hline \hline \multicolumn{3}{c|}{$G_{1 g}, 10 \times 10,24^{3} \times 64$} & & \multicolumn{2}{c}{$G_{1 g}, 10 \times 10,16^{3} \times 64$} \\
\hline Energy & $Z_{n}$ & Time & Energy & $Z_{n}$ \\
$0.193(3)$ & $5.75(15) \times 10^{-5}$ & $19-24$ & $0.194(4)$ & $8.45(15) \times 10^{-5}$ \\
$0.398(6)$ & $4.79(12) \times 10^{-5}$ & $8-12$ & $0.403(6)$ & $9.53(26) \times 10^{-5}$ \\
$0.399(7)$ & $5.64(33) \times 10^{-5}$ & $10-15$ & $0.409(8)$ & $8.84(46) \times 10^{-5}$ \\
& $G_{2 g}, 4 \times 4,24^{3} \times 64$ & & $G_{2 g}, 4 \times 4,16^{3} \times 64$ \\
Energy & $Z_{n}$ & Time & Energy & $Z_{n}$ \\
$0.374(13)$ & $1.47(57) \times 10^{-5}$ & $13-18$ & $0.414(13)$ & $2.11(35) \times 10^{-5}$ \\
$0.398(10)$ & $1.34(25) \times 10^{-5}$ & $10-14$ & $0.444(7)$ & $7.66(100) \times 10^{-5}$ \\
$0.574(21)$ & $3.73(30) \times 10^{-5}$ & $11-14$ & $0.564(12)$ & $5.90(199) \times 10^{-5}$ \\
& $H_{g}, 12 \times 12,24^{3} \times 64$ & & $H_{g}, 12 \times 12,16^{3} \times 64$ \\
Energy & $Z_{n}$ & Time & Energy & $Z_{n}$ \\
$0.365(9)$ & $5.40(15) \times 10^{-5}$ & $13-18$ & $0.375(9)$ & $9.25(33) \times 10^{-5}$ \\
$0.395(7)$ & $5.14(14) \times 10^{-5}$ & $10-15$ & $0.407(5)$ & $8.38(21) \times 10^{-5}$ \\
$0.400(7)$ & $4.39(7) \times 10^{-5}$ & $10-16$ & $0.407(8)$ & $7.75(10) \times 10^{-5}$ \\
$0.403(8)$ & $4.27(10) \times 10^{-5}$ & $10-14$ & $0.422(6)$ & $8.07(17) \times 10^{-5}$ \\
& & & $0.427(8)$ & $6.36(8) \times 10^{-5}$ \\
& & & $0.443(6)$ & $8.75(7) \times 10^{-5}$ \\
\hline \hline
\end{tabular}

TABLE VII. The $I=\frac{1}{2}, N^{*}$, negative-parity energies in the units of $a_{t}^{-1}$ obtained on a $24^{3} \times$ 64 lattice (left) and a $16^{3} \times 64$ lattice (right). A similar description applies as in the caption of Table VI.

\begin{tabular}{ccc|cc}
\hline \hline & $G_{1 u}, 7 \times 7,24^{3} \times 64$ & & \multicolumn{2}{c}{$G_{1 u}, 7 \times 7,16^{3} \times 64$} \\
\hline Energy & $Z_{n}$ & Time & Energy & $Z_{n}$ \\
$0.290(5)$ & $5.68(53) \times 10^{-5}$ & $12-17$ & $0.290(7)$ & $9.19(40) \times 10^{-5}$ \\
$0.323(12)$ & $5.73(44) \times 10^{-5}$ & $11-17$ & $0.340(6)$ & $9.35(73) \times 10^{-5}$ \\
& $G_{2 u}, 3 \times 3,24^{3} \times 64$ & & $G_{2 u}, 3 \times 3,16^{3} \times 64$ \\
Energy & $Z_{n}$ & Time & Energy & $Z_{n}$ \\
$0.327(10)$ & $1.93(82) \times 10^{-5}$ & $15-20$ & $0.325(9)$ & $2.67(121) \times 10^{-5}$ \\
$0.473(11)$ & $7.24(22) \times 10^{-6}$ & $7-12$ & & $H_{u}, 8 \times 8,16^{3} \times 64$ \\
& $H_{u}, 8 \times 8,24^{3} \times 64$ & & Energy & $Z_{n}$ \\
Energy & $Z_{n} \times 10^{-5}$ & $18-23$ & $0.284(9)$ & $8.07(26) \times 10^{-5}$ \\
$0.284(16)$ & $\left.5.00(38) \times 10^{-5}\right)$ & $0.310(8)$ & $1.04(1) \times 10^{-4}$ \\
$0.308(7)$ & $5.74(8) \times 10^{-5}$ & $15-22$ & $0.328(8)$ & $1.44(1) \times 10^{-5}$ \\
$0.330(9)$ & $7.93(5) \times 10^{-5}$ & $16-22$ & \multicolumn{2}{c}{} \\
\hline \hline
\end{tabular}


TABLE VIII. The $I=\frac{3}{2}, \Delta^{*}$, positive-parity energies in the units of $a_{t}^{-1}$ obtained on the $24^{3} \times$ 64 lattice (left) and the $16^{3} \times 64$ lattice (right). A similar description applies as in the caption of Table VI.

\begin{tabular}{ccc|cc}
\hline \hline & $G_{1 g}, 5 \times 5,24^{3} \times 64$ & & \multicolumn{2}{c}{$G_{1 g}, 5 \times 5,16^{3} \times 64$} \\
\hline Energy & $Z_{n}$ & Time & Energy & $Z_{n}$ \\
$0.378(17)$ & $5.05(14) \times 10^{-5}$ & $14-18$ & $0.373(8)$ & $1.67(5) \times 10^{-4}$ \\
$0.392(7)$ & $7.41(26) \times 10^{-5}$ & $12-17$ & $0.401(13)$ & $9.33(16) \times 10^{-5}$ \\
& $G_{2 g}, 1 \times 1,24^{3} \times 64$ & & $G_{2 g}, 1 \times 1,16^{3} \times 64$ \\
Energy & $Z_{n}$ & Time & Energy & $Z_{n}$ \\
$0.416(7)$ & $8.92(28) \times 10^{-5}$ & $9-14$ & $0.429(6)$ & $1.49(4) \times 10^{-4}$ \\
& $H_{g}, 4 \times 4,24^{3} \times 64$ & & $H_{g}, 4 \times 4,16^{3} \times 64$ \\
Energy & $Z_{n}$ & Time & Energy & $Z_{n}$ \\
$0.231(3)$ & $1.22(3) \times 10^{-4}$ & $19-26$ & $0.232(4)$ & $1.79(4) \times 10^{-4}$ \\
$0.326(7)$ & $5.89(5) \times 10^{-5}$ & $13-20$ & $0.341(13)$ & $1.11(1) \times 10^{-4}$ \\
$0.370(25)$ & $6.54(4) \times 10^{-5}$ & $19-23$ & $0.399(10)$ & $1.20(6) \times 10^{-4}$ \\
$0.400(8)$ & $7.15(5) \times 10^{-5}$ & $14-19$ & $0.402(6)$ & $1.29(2) \times 10^{-4}$ \\
\hline \hline
\end{tabular}

TABLE IX. The $I=\frac{3}{2}, \Delta^{*}$, negative-parity energies in the units of $a_{t}^{-1}$ obtained on the $24^{3} \times$ 64 lattice (left) and the $16^{3} \times 64$ lattice (right). A similar description applies as in the caption of Table VI.

\begin{tabular}{ccc|cc}
\hline \hline & $G_{1 u}, 6 \times 6,24^{3} \times 64$ & & \multicolumn{2}{c}{$G_{1 u}, 6 \times 6,16^{3} \times 64$} \\
\hline Energy & $Z_{n}$ & Time & Energy & $Z_{n}$ \\
$0.309(9)$ & $3.13(99) \times 10^{-6}$ & $15-20$ & $0.312(7)$ & $5.38(117) \times 10^{-5}$ \\
& $G_{2 u}, 1 \times 1,24^{3} \times 64$ & & $G_{2 u}, 1 \times 1,16^{3} \times 64$ \\
Energy & $Z_{n}$ & Time & Energy & $Z_{n}$ \\
$0.506(7)$ & $7.31(16) \times 10^{-5}$ & $6-12$ & $0.539(5)$ & $1.43(2) \times 10^{-4}$ \\
& $H_{u}, 7 \times 7,24^{3} \times 64$ & & $H_{u}, 7 \times 7,16^{3} \times 64$ \\
Energy & $Z_{n}$ & Time & Energy & $Z_{n}$ \\
$0.320(5)$ & $8.38(30) \times 10^{-5}$ & $12-17$ & $0.317(6)$ & $1.16(4) \times 10^{-4}$ \\
$0.485(7)$ & $5.56(53) \times 10^{-5}$ & $7-12$ & $0.485(8)$ & $9.14(28) \times 10^{-5}$ \\
\hline \hline
\end{tabular}

with $I=\frac{3}{2}$, and negative-parity states with $I=\frac{3}{2}$, respectively. Operators are selected so as to provide the best quality of energies for the larger lattice volume and the same sets of operators are then used for the smaller lattice volume. The $Z$ factors given in Eq. (25) are also shown along with the energies. Each table shows results for two lattices, $16^{3} \times 64$ and $24^{3} \times 64$, and six symmetry channels, $G_{1 g / u}, G_{2 g / u}$ and $H_{g / u}$. In some channels, there are states in the $16^{3} \times 64$ analysis that are missing from the $24^{3} \times 64$ analysis and vice versa.

We have examined the $Z$ factors defined in Eqs. (25) and (26) as a possible means to detect scattering states following the methods of Ref. [26]. This has not produced clearly interpretable results. Therefore we discuss the results for each channel in the following subsections assuming that there are no scattering states in the extracted spectra for the pion mass used in these calculations.

\section{A. $G_{1}$ channel}

In the $I=\frac{1}{2}, G_{1 g}$ channel, we selected 10 operators on the $24^{3} \times 64$ lattice that give the best quality of energies using the variational method, and the same 10 operators are used to analyze the $16^{3} \times 64$ lattice data. The ground state of this channel, i.e., the nucleon state, has a very stable set of eigenvector components with respect to time as shown in Fig. 3. As many studies have shown, the ground state is dominated by a local operator of the form $u^{T}\left(C \gamma_{5}\right) d P_{+} u$, where $P_{ \pm}$are parity projection matrices for Dirac spinors. However, we find non-negligible contributions from nonlocal $T_{1}$ operators represented by diamonds, circles, and inverted triangles in the figure. One significant operator has a mixed-symmetric isospin symmetry with totally symmetric Dirac indices. Although this isospin symmetry is unusual, nonlocal operators formed from three quarks allow such combinations. In fact, this operator plays a significant role in the first excited state in combination with a local operator of the second embedding. The first and second excited states have relatively clean signals and are dominated by local and $T_{1}$-displaced operators, respectively. Similar energies are calculated for states on the two lattice volumes except for the second excited state. Dominance of a nonlocal operator in the second-excited-state eigenvector 


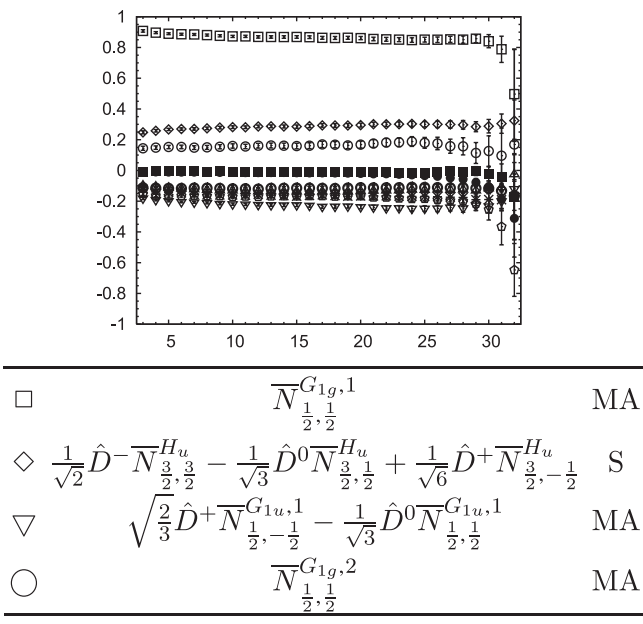

(a) Nucleon, ground state

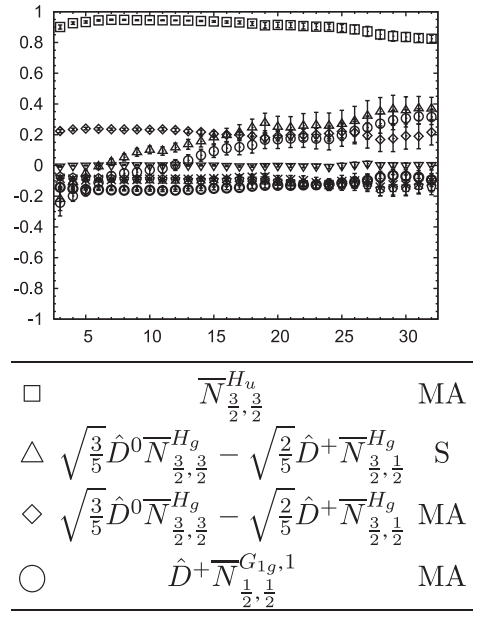

(b) $N^{*}$, lowest $H_{u}$ state

FIG. 3. Eigenvector components $v_{k}^{(0)}(t, 0)$ of the ground state for (a) the $I=\frac{1}{2}, G_{1 g}$ channel using a $10 \times 10$ matrix and (b) the $I=\frac{1}{2}$, $H_{u}$ channel using an $8 \times 8$ matrix, both on the $24^{3} \times 64$ lattice. The vertical axis is the value of the coefficient of a basis operator and the horizontal axis is time. Each basis operator corresponds to a symbol in the plot; the four most important operators are listed below the plot together with their symbols. $\bar{N}_{S, S_{z}}^{\lambda k}$ is our notation for a smeared three-quark operator with spin $S$, spin projection $S_{z}$, irrep $\Lambda$, and embedding $k$. The symmetry (MA, MS, or S) of Dirac indices for each three-quark operator is written next to the operator. Explicit forms of the operators $\bar{N}_{S, S_{2}}^{\lambda k}$ are provided in Ref. [17].

might explain this dependence on lattice volume because we find that the nonlocal operators generally exhibit more sensitivity to the spatial volume. Eigenvectors are more or less the same in each state on the two lattices as far as the coefficients of the two or three most important operators are concerned. Fluctuations of the eigenvector components generally increase with time for excited states, but they are fairly stable in the time ranges where energies are extracted.

In the $G_{1 u}$ channel we obtain two states which could correspond to $N\left(\frac{1}{2}^{-}, 1535\right)$ and $N\left(\frac{1}{2}^{-}, 1650\right)$. Each is dominated by two local operators but in different linear combinations. Contributions from nonlocal operators for the first excited state are around $\left|v_{k}^{(n)}(t, 0)\right| \lesssim 0.3$, which is less than for positive parity states. The energy of the lowest state on the two lattice volumes is essentially the same, suggesting that a $16^{3}$ lattice is large enough to contain this state with a $490 \mathrm{MeV}$ pion mass.

In the $I=\frac{3}{2}, G_{1 g}$ channel, the first excited (the lowest) state in $16^{3}$ volume corresponds to the lowest (the first excited) state in $24^{3}$ volume. This correspondence is based on the similarity of eigenvector components. The first excited state of $I=\frac{3}{2}, G_{1 g}$ has lattice energy of $0.392(7)$ on the larger volume and $0.373(8)$ on the smaller volume, with error bars that do not overlap. The apparent crossover of energies of the same state at different lattice volumes is observed only in this particular state. The eigenvectors for these states show that they are dominated by $T_{1}$ displaced operators with MS isospin.

In the negative parity channel, it is more difficult to determine the excited state energies due to the large error bars, while the lowest state, corresponding to $\Delta\left(\frac{1}{2}^{-}, 1620\right)$, is fairly stable. Again the $T_{1}$ displaced operators dominate the eigenvectors of the two lowest energy states.

\section{B. $H$ channel}

We find a richer spectrum in $I=\frac{1}{2}, H_{g / u}$ than in other channels as shown in Tables VI, VII, and VIII. Plateaus are achieved for $t \approx 20$ in the first three states. Approximate plateaus could be selected for the next three states. However, the use of more diverse operators together with better statistics is required in order to extract reliably more than the first three states.

We obtained four energies for the $I=\frac{1}{2}, H_{g}$ channel using the $24^{3}$ lattice and six energies using the $16^{3}$ lattice, with errors relatively small compared with those in other channels. A quasilocal operator yields the dominant coupling to the lowest $H_{g}$ state and our operator is essentially the same as the Rarita-Schwinger projected operator that has been used in other works. The Rarita-Schwinger projected lattice interpolating field belongs to the $H$ irrep and is therefore consistent with the subduction of continuum spins $\frac{3}{2}, \frac{5}{2}, \frac{7}{2}, \cdots$. Several works $[4,6,7,9]$ have suggested that this lowest $H_{g}$ state likely corresponds to a continuum spin $\frac{3}{2}^{+}$state. However, we find an indication that it corresponds to a higher spin, either $\frac{5}{2}^{+}$as in the $N\left(\frac{5}{2}^{+}, 1680\right)$, or $\frac{7}{2}^{+}$. The possible spins will be discussed in the next section when the patterns of states over all the irreps are considered.

For states above the ground state, combinations of nonlocal $T_{1}$ operators dominate the couplings. Consistent with this, we observe a finite volume effect on some energies in 
the $N^{*}, H_{g}$ excited spectrum. Even though the lowest energy state is dominated by the quasilocal operator, its energy has some dependence on the volume of the lattice. The lowest and the first excited states are clearly identifiable from eigenvector compositions in both volumes. Eigenvectors for the second and the third excited states are contaminated by noise on the $24^{3}$ lattice with the available statistics, although the $16^{3}$ lattice provides stable eigenvectors.

The $I=\frac{1}{2}, H_{u}$ states provide the least contaminated group of states in the sense that energies for several excited states are determined easily and the lowest few states have eigenvectors that are nearly as stable as those of the nucleon $G_{1 g}$ ground state. The lowest-lying state should correspond to the physical $N\left(\frac{3}{2}-1520\right)$ state, the first excited state should correspond to the $N\left(\frac{5}{2}^{-}, 1675\right)$ state, and the second excited state should correspond to the $N\left(\frac{3}{2}^{-}, 1700\right)$ state, supposing no scattering states are involved. Energy differences between these states are relatively small and our lattice results agree with this pattern. In contrast to the positive parity channel, finite volume effects in energies are well within statistical fluctuations. The nonlocal three-quark operators that we use yield nonvanishing MS isospin combinations although the simplest quark model does not have them. Our results show that contributions of MS isospin operators are crucial to the first and second $H_{u}$ excited states. The same operators are important in both volumes. Energy differences between parity-partner states are found to be smaller for the $I=$ $\frac{1}{2}, H$ irreps than for other channels.

The $\Delta$ baryon appears as the lowest energy state in the $I=\frac{3}{2}, H_{g}$ channel. A local operator with nonrelativistic spin components dominates the ground state with very small error bars. The first and second excited state signals should correspond to $\Delta\left(\frac{3}{2}^{+}, 1600\right)$ and $\Delta\left(\frac{5}{2}^{+}, 1905\right)$, respectively. Signals for these states are also reasonably clean on the $16^{3}$ lattice, however the results on the $24^{3}$ lattice are subject to larger fluctuations and the extracted energies are not reliable.

In the $I=\frac{3}{2}, H_{u}$ spectrum we find that the ground state, corresponding to $\Delta\left(\frac{3}{2}-1700\right)$, has stable signatures in both energy and eigenvector plots but the signal is short-lived. This is associated with the fact that the most important operator is the negative parity transform of the operator that dominates the positive parity ground state. The resulting long-lived plateau in the backward-in-time part of the correlation function limits the range of times where the excited state energies can be extracted. The energy differences between parity-partner states of the low-lying delta baryon spectrum in the $H$ irrep are larger than in other channels.

\section{C. $G_{2}$ channel}

The $G_{2}$ baryon spectrum is the least explored because nonlocal operators have not been readily available.
However, $G_{2}$ operators are very important for the assignment of higher spins because of their role in the patterns of subduction of continuum spins to the octahedral irreps.

We have selected the four best operators for the $I=\frac{1}{2}$, $G_{2 g}$ spectrum by the method described earlier. One of these is the operator with $E$-type spatial displacement, which transforms evenly under spatial inversion. We find that $G_{2}$ energies are the most difficult to extract in our calculations because the plateaus are relatively short-lived, signal-tonoise ratios are small, and the number of operators that we have used for the variational method is limited. The limitation on the number of operators arises because we have restricted the operators to ones that can be constructed from one-link displacements. Work in progress includes more varied types of operators, which yield larger numbers of $G_{2}$ operators [11].

The lowest state in the $I=\frac{1}{2}, G_{2 g}$ channel has significant couplings to the $T_{1}$-displaced MA isospin operator, the $T_{1}$-displaced MS isospin operator, and the $E$-displaced MS isospin operator. There is a non-negligible contribution to the lowest energy state of $G_{2}$ from the $E$-type of displaced operator that corresponds to $L \geq 2$ in the subduction of continuum angular momenta. The first excited state has significant couplings to a combination of two $T_{1}$ operators that also contribute to the ground state but in a different linear combination. The second excited state couples almost purely to a single $T_{1}$ operator with MS isospin. Eigenvectors are similar on the two lattice volumes for the lowest three states, however their energies change significantly. The lowest energy is calculated to be $0.362(17)$ in this channel, which agrees very well with the lowest $I=\frac{1}{2}, H_{g}$ energy, 0.365(9). Because these two energies are degenerate within errors, they are consistent with being the partner states for spin $\frac{5}{2}$. However, that is not the only possibility as is discussed in the next section.

For the $I=\frac{1}{2}, G_{2 u}$ channel, the $T_{1}$-displaced operator with MS isospin dominates the lowest state, while the $E$-displaced operator plays a significant role with $\left|v_{k}^{(n)}(t, 0)\right| \sim 0.4$ in the first excited state. The lowest physical state corresponding to $I=\frac{1}{2}, G_{2 u}$ is $N\left(\frac{5}{2}^{-}, 1675\right)$, which should also occur as the first excited state of the $H_{u}$ spectrum. The lowest energy state of $H_{u}$ should correspond to $N\left(\frac{3}{2}^{-}, 1520\right)$. We find that the first excited state of the $H_{u}$ channel has energy 0.308(7), whereas the lowest state $G_{2 u}$ state has energy $0.327(10)$ in the larger volume. In the smaller lattice volume, $H_{u}$ and $G_{2 u}$ energies are 0.310(8) and $0.325(9)$, respectively. The possible spin assignments are discussed further in the next section.

Having several good operators in each symmetry channel is important for the success of the variational method. Based on our present results using four $G_{2}$ operators, we find that the energy of $I=\frac{1}{2}, G_{2 u}$ is lighter than that of $I=$ $\frac{1}{2}, G_{2 g}$, which is also consistent with experiment. An earlier analysis with one $G_{2}$ operator found the opposite [8]. 
For the $I=\frac{3}{2}, G_{2}$ channels, our set of quasilocal and one-link-displaced operators does not contain a subset that provides better signals than are obtained from a correlation function based on a single operator. The problem is noise. Therefore we simply fit the energies based on individual correlation functions and pick the best-behaved one, which is found to involve the $T_{1}$-displaced operator. Because no diagonalization is involved, the energy is relatively contaminated in early time slices and the plateau is weak. The $I=\frac{3}{2}, G_{2}$ spectrum needs further study using a larger set of operators, such as two-link-displaced operators or operators that have two quarks displaced from the third quark.

\section{PATTERN OF LOWEST-LYING ENERGIES}

The physical spectrum of baryon excited states shows a number of degeneracies between states of different spins. In the lattice results, this means that particular care must be exercised in order to identify spins because accidental degeneracies of two states can provide the same patterns in the octahedral irreps as a single higher spin state. Given the limited energy resolution of our calculations, this leads to alternative interpretations for the spins of some excited states in the lattice results. It is more appropriate to compare the pattern of lattice results to the pattern of experimental masses and spins subduced to the irreps of the octahedral group.

Although there are substantial discretization errors with the quark action that is used, and they could contribute differently in the different irreps, clear patterns in the degeneracies emerge. Focusing on the group of three positive-parity excited states near lattice energy $a_{t} E=$ 0.36 in Fig. 4, two interpretations are possible. (a) The

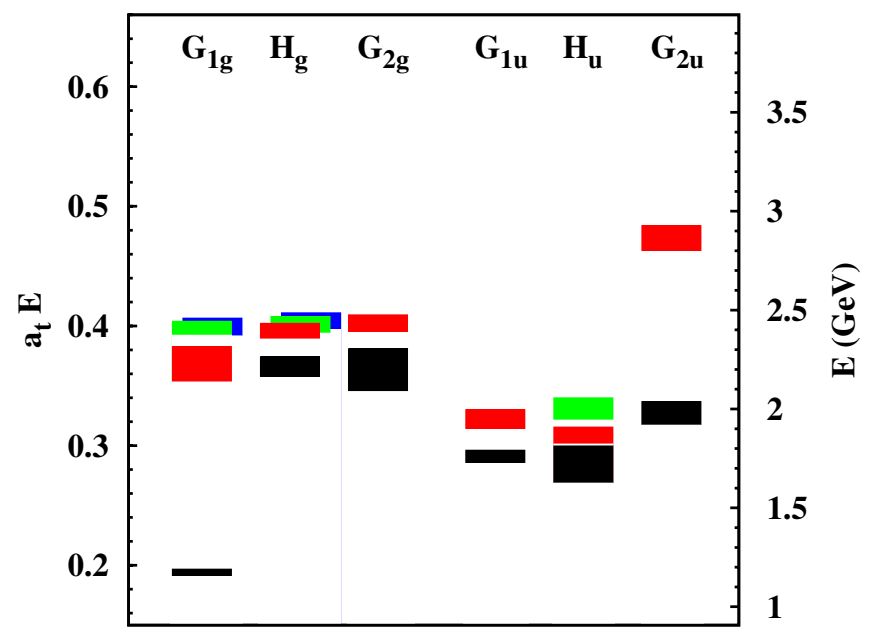

FIG. 4 (color online). The energies obtained for each symmetry channel of $I=\frac{1}{2}$ baryons are shown based on the $24^{3} \times 64$ lattice data. The scale on the left side shows energies in lattice units and the scale on the right side shows energies in $\mathrm{GeV}$. The scale was set using the string tension. Errors are indicated by the vertical size of the box. group consists of a spin $\frac{1}{2}$ state and a spin $\frac{5}{2}$ state that accidentally are degenerate. In this case the $G_{1 g}$ state corresponds to spin $\frac{1}{2}$ and the $H_{g}$ and $G_{2 g}$ partner states correspond to the subduction of spin $\frac{5}{2}$. (b) The group consists of a single state with the degenerate $G_{1 g}, H_{g}$ and $G_{2 g}$ partner states corresponding to the subduction of spin $\frac{7}{2}$. Note that these are the only possible interpretations. It is not possible for the $H$ state to be an isolated spin $\frac{3}{2}$ state because that would require the $G_{2}$ state also to be an isolated state. There is no interpretation for an isolated $G_{2}$ state. Higher spins than $\frac{7}{2}$ would require more partner states than are found.

In the physical spectrum of positive-parity nucleon resonances, the lowest excited state, $N\left(\frac{1}{2}^{+}, 1440\right)$, lies below all negative parity states. We do not find a signal for a positiveparity excitation that has lower energy than the negativeparity excitations at this quark mass. The next two excited nucleon states are essentially degenerate, namely, $N\left(\frac{5}{2}+1680\right)$ and $N\left(\frac{1}{2}^{+}, 1710\right)$, each with a width of about $100 \mathrm{MeV}$. Spin $\frac{7}{2}$ states occur only at significantly higher energy $(1990 \mathrm{MeV})$. These states are well separated from other states and the width of each is about $100 \mathrm{MeV}$. Primarily because of the absence of spin $\frac{7}{2}$ in the low-lying spectrum, interpretation (a) of our lattice results is more consistent with the pattern of physical energies and spins.

In the negative-parity spectrum, we also obtain essentially the same results for both lattice volumes. The three lowest states shown on the right half of Fig. 4 are unambiguously identified as follows: the lowest $G_{1 u}$ state corresponds to spin $\frac{1}{2}$ and the lowest two $H_{u}$ states correspond to distinct spin $\frac{3}{2}$ states. Above these is a group of three states with roughly the same lattice energy: $a_{t} E \approx 0.33$ (within errors). Again there are two possible interpretations. (a) The group consists of a spin $\frac{1}{2}$ state in $G_{1 u}$ that is accidentally degenerate with a spin $\frac{5}{2}$ state, the latter having degenerate partner states in $H_{u}$ and $G_{2 u}$. (b) The group consists a spin $\frac{7}{2}$ state having degenerate partner states in $G_{1 u}, H_{u}$ and $G_{2 u}$.

The pattern of low-lying physical states starts with $N\left(\frac{3}{2}{ }^{-}, 1520\right), N\left(\frac{1}{2}^{-}, 1535\right)$ and $N\left(\frac{3}{2}-1700\right)$. These should correspond to distinct $H_{u}, G_{1 u}$ and $H_{u}$ states on the lattice, in agreement with the three lowest negative-parity states in Fig. 4. The next two physical states are $N\left(\frac{1}{2}^{-}, 1680\right)$ and $N\left(\frac{5}{2}-1675\right)$, which essentially are degenerate. They should show up as degenerate $G_{1 u}, H_{u}$ and $G_{2 u}$ states on the lattice. This pattern of spins is consistent with interpretation (a) of the lattice states at lattice energy $a_{t} E \approx 0.33$. The pattern of energies of the physical states has $N\left(\frac{5}{2}^{-}, 1675\right)$ a little lower in energy than $N\left(\frac{3}{2}^{-}, 1700\right)$, but the lattice results at lattice spacing $0.1 \mathrm{fm}$ place the spin $\frac{5}{2}$ state above the spin $\frac{3}{2}$ state. Study of the continuum limit of the lattice spectrum is required in order to resolve these issues. 


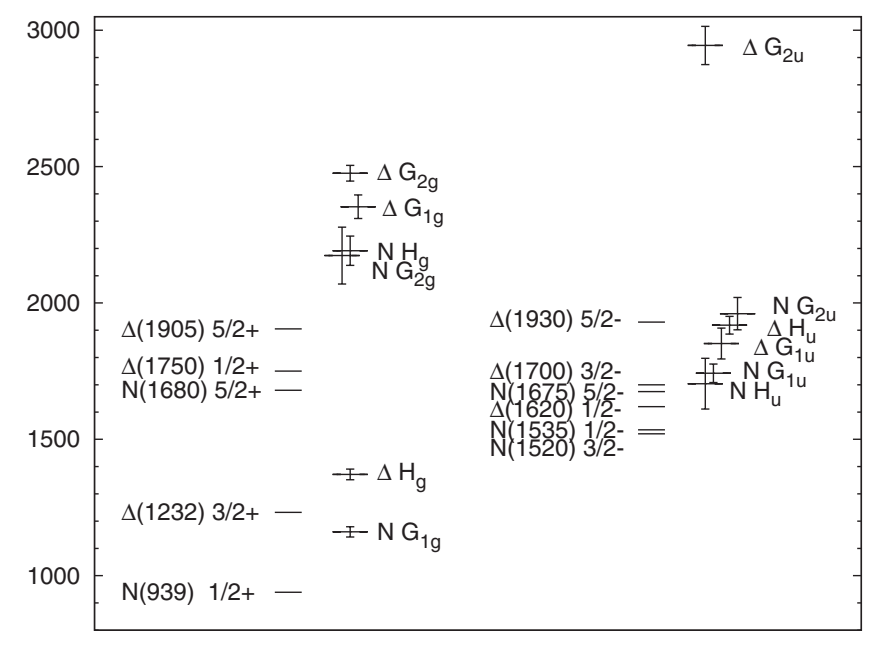

FIG. 5. The lowest energies obtained for each symmetry channel of $I=\frac{1}{2}$ and $I=\frac{3}{2}$ baryons are shown in columns 2 and 4 and the experimental levels are shown in columns 1 and 3 . The $24^{3} \times$ 64 lattice data are shown and the left two columns show the energies of positive-parity states and the right two columns show the energies of negative-parity states. The vertical axis shows energy in $\mathrm{MeV}$.

Figure 5 shows the pattern of lowest-lying energies for each irrep with the $\Delta$ states included. Energies have been converted to physical units but the purpose of this graph is to display the pattern of level orderings for low lying spinparity channels with spins up to $\frac{5}{2}$, not to make a comparison of absolute energy values with experiment.

The spin assignments in the negative parity channels obtained in our simulations correspond reasonably well with the physical spectrum, i.e., the $N\left(\frac{3}{2}^{-}\right)$is the lowest, the $N\left(\frac{1}{2}^{-}\right)$is slightly above, the $\Delta\left(\frac{1}{2}^{-}\right)$is next, the $\Delta\left(\frac{3}{2}^{-}\right)$is next, the $N\left(\frac{5}{2}^{-}\right)$, to which $I=\frac{1}{2}, G_{2 u}$ corresponds, is slightly above, and finally the $\Delta\left(\frac{5}{2}^{-}\right)$is highest. One result that does not correspond with nature is the relative order of the $N\left(\frac{5}{2}^{-}\right)$and the $\left(\Delta_{2^{-}}^{-}\right)$states, although the error bars overlap and the energy splitting of the mean values is only $2 \%$. Considering these six states, the mean energies of $N\left(\frac{3}{2}-\right)$ and $N\left(\frac{1}{2}^{-}\right)$are less than the other four. This agrees with what is predicted by the quark model with the contact spin-spin interaction between quarks treated as a perturbation.

In the positive-parity channels, with the exception of the Roper resonance, our spin assignments also follow the same orderings as occur in nature: the $N\left(\frac{1}{2}^{+}\right)$is the lowest, the $\Delta\left(\frac{3}{2}^{+}\right)$is next, the $N\left(\frac{5}{2}^{+}\right)$is next, for which $H_{g}$ and $G_{2 g}$ are degenerate, the $\Delta\left(\frac{1}{2}^{+}\right)$is next up, and finally the $\Delta\left(\frac{5}{2}^{+}\right)$ is highest. The analysis of Isgur and Karl based on the quark model yields a nearly degenerate set of states corresponding to $N\left(\frac{1}{2}^{+}, 1710\right), N\left(\frac{3}{2}^{+}, 1720\right)$, and $N\left(\frac{5}{2}^{+}, 1680\right)$. Their calculation explains well the small energy splitting, $\simeq 40 \mathrm{MeV}$, between these states. Our results yield a fair agreement with this result. Energies of the first excited state of $I=\frac{1}{2}, G_{1 g}$, the first excited state of $I=\frac{1}{2}, H_{g}$, and the lowest $I=\frac{1}{2}, G_{2 g}$ are 0.398(6), 0.395(7), and $0.362(17)$, respectively. Closeness of first two energies above is remarkable, but the third energy is significantly different. Note that our assignments cause the lowest $N\left(\frac{3}{2}^{+}\right)$ to correspond to the first excited state of $I=\frac{1}{2}, H_{g}$ because the lowest state corresponds to the $J^{P}=\frac{5}{2}^{+}$. Although our energy splittings at this quark mass can be much larger than those in the physical spectrum, if our spin assignments are correct the ordering of energies of the lightest states in a given channel reproduces the physical ordering.

On the other hand, comparison of the order of energy levels with different parities provides different conclusions. For instance, the $\Delta\left(\frac{3}{2}^{-}\right)$energy should appear between $N\left(\frac{5}{2}^{+}\right)$and $N\left(\frac{3}{2}^{+}\right)$energies (here only the lowest energies are considered), but it does not. Further study of the continuum limit is required in order to confirm the spin assignments.

\section{SUMMARY AND OUTLOOK}

This paper presents spectra for isospin $I=\frac{1}{2}$ and $I=\frac{3}{2}$ states based on lattice QCD simulations using the quenched approximation, anisotropic lattices with $a_{s} / a_{t}=$ 3 and a pion mass of $490 \mathrm{MeV}$. Sets of three-quark interpolating fields are used to construct matrices of correlation functions and the variational method is used to extract energies. Smeared quark and gluon fields are used to diminish the coupling to short wavelength fluctuations of lattice QCD. The result is that diagonalizations are able to extract good signals for low-lying states. We have obtained as many as 17 energies for $I=\frac{1}{2}$ states and 10 energies for $I=\frac{3}{2}$ baryon states, including various spin-parity channels. The variational method determines the best linear combinations of basis operators for energy eigenstates. For each obtained energy eigenstate, we paid close attention to the stability of the eigenvector components with respect to time and with respect to the two different volumes.

Because scattering states are expected to be present in the spectrum, we have calculated spectral weights for all states on two volumes. However, the weights do not provide evidence for two-particle states in our spectra. The first positive-parity excited state in the $I=\frac{1}{2}, G_{1 g}$ channel is found to have energy significantly higher than the experimentally known mass of the Roper resonance. Better ways are being developed to identify scattering states and resonances, such as the use of operators that should have large couplings to the $\pi-N$ states and the use of several pion masses in order to better detect $s$-wave $\pi N$ states.

Because the minimum spin that is contained in the $G_{2}$ irrep is $\frac{5}{2}$, we have found strong evidence for spin $\frac{5}{2}$ or higher for both parities in our spectra for $I=\frac{1}{2}$. We also have found evidence for degenerate partner states corresponding to the subduction of spin $\frac{5}{2}$ or higher to the octahedral irreps. 
The lattice results for the $I=\frac{1}{2}$ low-lying excited states provide the correct number and pattern of octahedral states for the subduction of the spins of the low-lying physical states, with the exception of the Roper state. When the $\Delta$ states are included, the overall pattern of lattice results for the lowest energy in each channel is similar to the pattern observed in nature.

The results shown here and in Ref. [11] represent a first glimpse of the pattern of nucleon and $\Delta$ excitations as predicted by QCD, but they are based on the quenched approximation, a $490 \mathrm{MeV}$ pion mass and an action that may have significant discretization effects. Similar calculations in full QCD are under way with an improved action, several pion masses, several lattice spacings and much larger sets of baryon interpolating operators.

\section{ACKNOWLEDGMENTS}

This work was supported by the U.S. National Science Foundation under Grant No. PHY-0653315, and by the U.S. Department of Energy under Contracts No. DEAC05-84ER40150 and No. DE-FG02-93ER-40762.
[1] S. Aoki et al. (CP-PACS Collaboration), Phys. Rev. Lett. 84, 238 (2000).

[2] C. T. H. Davies et al. (HPQCD Collaboration), Phys. Rev. Lett. 92, 022001 (2004).

[3] A. Ali Khan et al. (CP-PACS Collaboration), Phys. Rev. D 65, 054505 (2002); 67, 059901(E) (2003).

[4] L. Zhou and F. X. Lee, Phys. Rev. D 74, 034507 (2006).

[5] T. Burch, C. Gattringer, L. Y. Glozman, C. Hagen, D. Hierl, C. B. Lang, and A. Schafer, Phys. Rev. D 74, 014504 (2006).

[6] K. Sasaki and S. Sasaki, Proc. Sci., LAT2005 (2005) 060.

[7] K. Sasaki and S. Sasaki, Phys. Rev. D 72, 034502 (2005).

[8] S. Basak et al. (LHP Collaboration), Nucl. Phys. B, Proc. Suppl. 140, 278 (2005).

[9] J. M. Zanotti, D. B. Leinweber, A. G. Williams, J. B. Zhang, W. Melnitchouk, and S. Choe (CSSM Lattice Collaboration), Phys. Rev. D 68, 054506 (2003).

[10] D. Guadagnoli, M. Papinutto, and S. Simula, Phys. Lett. B 60474 (2004).

[11] A. Lichtl, arXiv:hep-lat/0609019.

[12] M. Albanese et al. (APE Collaboration), Phys. Lett. B 192, 163 (1987).

[13] M. G. Alford, T. Klassen, and P. Lepage, Nucl. Phys. B, Proc. Suppl. 47, 370 (1996).
[14] A. Lichtl, S. Basak, R. Edwards, G. T. Fleming, U. M. Heller, C. Morningstar, D. Richards, I. Sato, and S. J. Wallace, Proc. Sci., LAT2005 (2005) 076.

[15] C. Michael, Nucl. Phys. B259, 58 (1985).

[16] M. Lüscher and U. Wolff, Nucl. Phys. B339, 222 (1990).

[17] S. Basak et al. (Lattice Hadron Physics Collaboration), Phys. Rev. D 72, 074501 (2005).

[18] S. Basak et al., Phys. Rev. D 72, 094506 (2005).

[19] S. Capstick and W. Roberts, Prog. Part. Nucl. Phys. 45, S241 (2000).

[20] N. Isgur and G. Karl, Phys. Rev. D 19, 2653 (1979); 23, 817(E) (1981).

[21] P. Chen, Phys. Rev. D 64, 034509 (2001).

[22] T. R. Klassen, Nucl. Phys. B533, 557 (1998).

[23] F. Karsch, J. Engels, and T. Scheideler, Nucl. Phys. B, Proc. Suppl. 63, 427 (1998).

[24] M. Fujisaki et al. (QCD-TARO Collaboration), Nucl. Phys. B, Proc. Suppl. 53, 426 (1997).

[25] K. G. Wilson, Phys. Rev. D 10, 2445 (1974).

[26] N. Mathur, F. X. Lee, A. Alexandru, C. Bennhold, Y Chen, S. J. Dong, T. Draper, I. Horvath, K. F. Liu, S. Tamhankar, and J. B. Zhang, Phys. Rev. D 70074508 (2004). 